\title{
Principales factores que influyen en la selección de estudios superiores de los estudiantes de la Universidad de Los Lagos, sede Chiloé
}

\author{
Francisco Kroff Trujillo ${ }^{1}$ - Fabiola Sandoval Henríquez ${ }^{2}$ - Nicolás Iglesias Mills ${ }^{3}$
}

\begin{abstract}
Recepción: 11-12-2019 / Aceptación: 21-01-2020
\end{abstract}
\section{Resumen}

El trabajo desarrollado tiene como propósito conocer cuáles son los principales factores considerados por los estudiantes de la Universidad de Los Lagos sede Chiloé, Castro - Ancud, de toda la cohorte 2019 (todos los estudiantes de primer año), sobre la selección de una Institución de Educación Superior. Así como también, qué factores incidieron para escoger la carrera en la cual se matricularon: sociales, académicas, económicas y vinculares.

Se utilizó el paradigma explicativo, no experimental y cuantitativo con el convencimiento de lograr antecedentes lo más objetivos posibles. Se aplicó la técnica de la encuesta para la recogida y posterior insumo del análisis de datos cuantitativos.

Los resultados indican que el factor más importante por parte de los estudiantes es el acceso a las becas y beneficios que las instituciones de Educación Superior pueden entregar. Finalmente, se proponen diferentes acciones que se deben realizar, donde la principal es el fortalecimiento de vínculos con los Establecimientos Educacionales de la Provincia, para realizar actividades en ayuda de los estudiantes que egresan de sus aulas, como el acompañamiento de la obtención de becas o preparación a las pruebas de admisión general de las universidades chilenas.

Palabras clave: Educación Superior, selección de carrera, selección universitaria, contexto, Chiloé.

\begin{abstract}
The work carried out has the purpose to know what are the main factors considered by the students of the University of Los Lagos headquarters Chiloé, Castro - Ancud, of the entire cohort 2019 (all firstyear students) about the selection of a higher education institution. As well as, what factors influenced to select the major in which they enrolled: social, academic, economic, and bonding.

The explanatory, non-experimental and quantitative paradigm was used with the conviction of reaching the most objective background possible. The survey technique was applied for the collection and subsequent input of the analysis of quantitative data.

The results indicate that the most important factor on the part of the students is the access to the scholarships and benefits which the institutions of Higher Education can provide. Finally, different actions are proposed that have to be carried out, where the main one is the strengthening of bonds with the Educational Establishments of the Province, to perform activities in support of the students who leave from their classrooms, such as the accompaniment of obtaining scholarships or preparation for the general admission tests of Chilean universities.
\end{abstract}

Keywords: Higher education, major selection, university selection, context, Chiloé.

1. Magíster en el Modelado del Conocimiento para entornos educativos virtuales, Académico, Jefe de Carrera, Universidad de Los Lagos, Chile; email: francisco.kroff@ulagos.cl

2. Magíster en Gestión de Negocios, Encargada de Gestión y Aseguramiento de Calidad, Universidad de Los Lagos; email: fabiola.sandoval@ulagos.cl

3. Magíster en Educación, mención Políticas y Gestión, Investigador Independiente; email: nicolasimuy@gmail.com 


\section{Introducción}

El estudio se realizó en la Universidad de Los Lagos sede Chiloé, Castro - Ancud, Chile, con el objetivo de entender qué elementos son considerados por los estudiantes de dicha universidad para la elección de la misma. Se entiende que en un contexto como el de Chile, profundizado en la realidad del archipiélago de Chiloé, los factores a considerar para la elección de la universidad pueden ser únicos, propios del contexto que analizamos, y al mismo tiempo generalizable para otros estudios. Por supuesto, se entiende que estos podrían variar según región, pero se considera también que las particularidades de un territorio como Chiloé hacen que se vean representados elementos culturales y contextuales propios de características urbanas, periurbanas y rurales de diversas regiones de Chile, y del continente latinoamericano. $\mathrm{Al}$ mismo tiempo, la representatividad puede ser llevada al grupo de universidades insulares del mundo (propias de contextos de territorios en islas), que de por sí tienen características especiales. Por ejemplo, la existencia de la RETI (Red de Excelencia de Territorios Insulares).

Cabe recordar que el sistema de educación terciaria chilena es un modelo neoliberal, pagado y en el que se puede acceder a becas, pero que responde sobre todo al estudiante como recurso económico.

En Chile, la implementación del neoliberalismo comenzó más de una década antes que el resto de América Latina. En efecto, es bajo el régimen de Augusto Pinochet que se pone en práctica por primera vez en la región -y en el mundo- lo que más tarde se denominará el modelo neoliberal (Harvey, 2007). Y, en lo que nos interesa, es en Chile donde, tal vez, se da la expresión más pura del modelo educacional neoliberal. Progresivamente, este ha ido preconizando el culto a la eficiencia, generalizando la competencia en todos los niveles, instaurando la cultura de la evaluación y entronizando el mérito como modelo de justicia social (Slachevsky, 2015).

Dentro de estos dos grandes grupos (Institución de Educación Superior y carrera universitaria), los factores que se consideraron fueron: caracterización del estudiante, es decir, edad, comuna, género y si rindió la Prueba de Selección Universitaria, que solo aplica a los estudiantes que estudian carreras profesionales con licenciatura. Es necesario recordar que la Universidad de Los Lagos en Chiloé cuenta con carreras profesionales con licenciatura, pero mayoritariamente con carreras técnicas y profesionales sin licenciatura.

Al mismo tiempo, también se consideraron factores como los territoriales, teniendo en cuenta que la mayoría de los estudiantes no viven necesariamente en la ciudad donde se encuentra la universidad; culturales o de estilo de vida, referidos a los valores que la institución promueve o la vida socio política dentro de la misma; elementos académicos como la carrera, los profesores o el programa; y factores económicos como la posibilidad de trabajar y estudiar al mismo tiempo, entre otros. 
Todo esto permite tener una idea más amplia de cuál es la percepción de la universidad en el territorio chilote, y poder generar -a futuro- vínculos efectivos de relacionamiento con el medio y significativos para los estudiantes. Esto genera un beneficio para todos los actores de la comunidad educativa chilota.

\section{Metodología}

El estudio se realizó bajo un paradigma explicativo, no experimental y cuantitativo. El instrumento de recogida de información utilizado fue el guion de preguntas semiestructurado, mediante la técnica de la encuesta. Esta, según Hernández, Fernández y Baptista (2010, p.120) es definida como "el método de investigación capaz de dar respuestas a problemas tanto en términos descriptivos como de relación de variables, tras la recogida de información sistemática, según el diseño previamente establecido que asegure el rigor de la información obtenida”. De esta manera el instrumento puede ser utilizado en forma segura para entregar descripciones precisas de los objetos o variables en estudio; además de permitir detectar patrones y relaciones entre las características descriptivas, y establecer nexos entre los diferentes eventos o situaciones expuestas.

La encuesta se diseñó con la herramienta Formulario de Google Drive y se aplicó a los estudiantes de primer semestre de las carreras que imparte la Universidad de Los Lagos en la sede Chiloé, Castro - Ancud en Chile.
El instrumento estuvo compuesto de cuatro ítems, más un espacio introductorio sobre aspectos generales e información que permitiera caracterizar a los estudiantes. El primer ítem trató sobre los principales factores en la selección de una Institución de Educación Superior; el segundo, sobre los principales factores en la selección de la Universidad de Los Lagos; el ítem número tres, sobre los principales factores en la selección de una carrera en educación superior y, por último, sobre los principales factores que tuvieron al seleccionar la carrera en la cual se matricularon.

La población estuvo formada por estudiantes de todas las carreras de la sede Chiloé, Castro - Ancud de la cohorte 2019, la cual fue de 373 estudiantes. Se realizó el cálculo muestral en la página web: https://es.surveymonkey.com/ $\mathrm{mp} /$ sample-size-calculator/, con un nivel de confianza de un $95 \%$ y un margen de error de un $5 \%$, arrojando una muestra de total de 190 sujetos. El criterio de selección fue el ser estudiantes de primer año de la cohorte 2019 de la sede.

La encuesta fue respondida por el $52.28 \%$ de la población total de matriculados en la cohorte 2019 de la Universidad de Los Lagos sede Chiloé, Castro-Ancud, con un $80 \%$ y $20 \%$, respectivamente.

\section{Resultados}

Con respecto a los antecedentes, los estudiantes tuvieron que contestar cinco preguntas referidas a: sede donde se matriculó, carrera, 
rango etario, género. Para el caso, la muestra se dividió en $44.10 \%$ hombres y $55.90 \%$ mujeres; entre las edades de menores o iguales a 19 años (47.69\%), de 20 a 39 años (48.72\%) y mayores o iguales a 40 años (3.59\%). Todos ellos ubicados en las sedes Castro (80\%) y Ancud (20\%).

En la tabla uno, se observa la distribución según la carrera elegida.

\section{Selección de una Institución de Educación Superior}

En esta sección se consultó sobre la importancia de las opiniones, las fuentes de información y la Institución de Educación Superior. Respecto a las opiniones de diferentes actores, la decisión propia del estudiante fue el principal factor (75.38\%). En segundo grado de importancia, se encontró la opinión de familiares directos (50.26\%).

En cuanto a las fuentes de información, el sitio web de la Universidad se destacó como la principal opción con un $49.74 \%$; seguido de las ferias vocacionales y/o universitarias (36.92\%). Al consultarles sobre la Institución de Educación Superior, las becas y beneficios que estas poseen y a las cuales pueden acceder los estudiantes fue el aspecto más señalado (75.90\%); mientras que la acreditación institucional obtuvo un $72.31 \%$ (ver figura 1 ).

\section{Selección Universidad de Los Lagos}

El principal factor para escoger la Universidad de Los Lagos sede Chiloé, Castro - Ancud, fue la ubicación geográfica, con un $57.44 \%$; seguido de la acreditación institucional con $17.44 \%$ (ver figura 2).

Con respecto los factores que se tuvieron en cuenta a la hora de seleccionar la Universidad de Los Lagos como la Institución de Educación Superior, se trabajaron con tres variables: sociales, académicos e infraestructura (ver figura 3).

Tabla 1. Caracterización de los estudiantes encuestados

\begin{tabular}{|c|c|c|}
\hline Variable & Categoría & Porcentaje \\
\hline \multirow{4}{*}{ Carrera } & Técnico Universitario en Administración de Empresas & $27.18 \%$ \\
\cline { 2 - 3 } & Técnico Universitario en Educación Parvularia & $26.15 \%$ \\
\cline { 2 - 3 } & Técnico Universitario en Electricidad y Automatización & $12.31 \%$ \\
\cline { 2 - 3 } & Técnico Universitario en Informática & $10.77 \%$ \\
\cline { 2 - 3 } & Técnico Universitario en Construcción & $10.77 \%$ \\
\cline { 2 - 3 } & Técnico Deportivo Universitario & $8.72 \%$ \\
\cline { 2 - 3 } & Técnico Universitario en Contabilidad & $4.10 \%$ \\
\hline
\end{tabular}




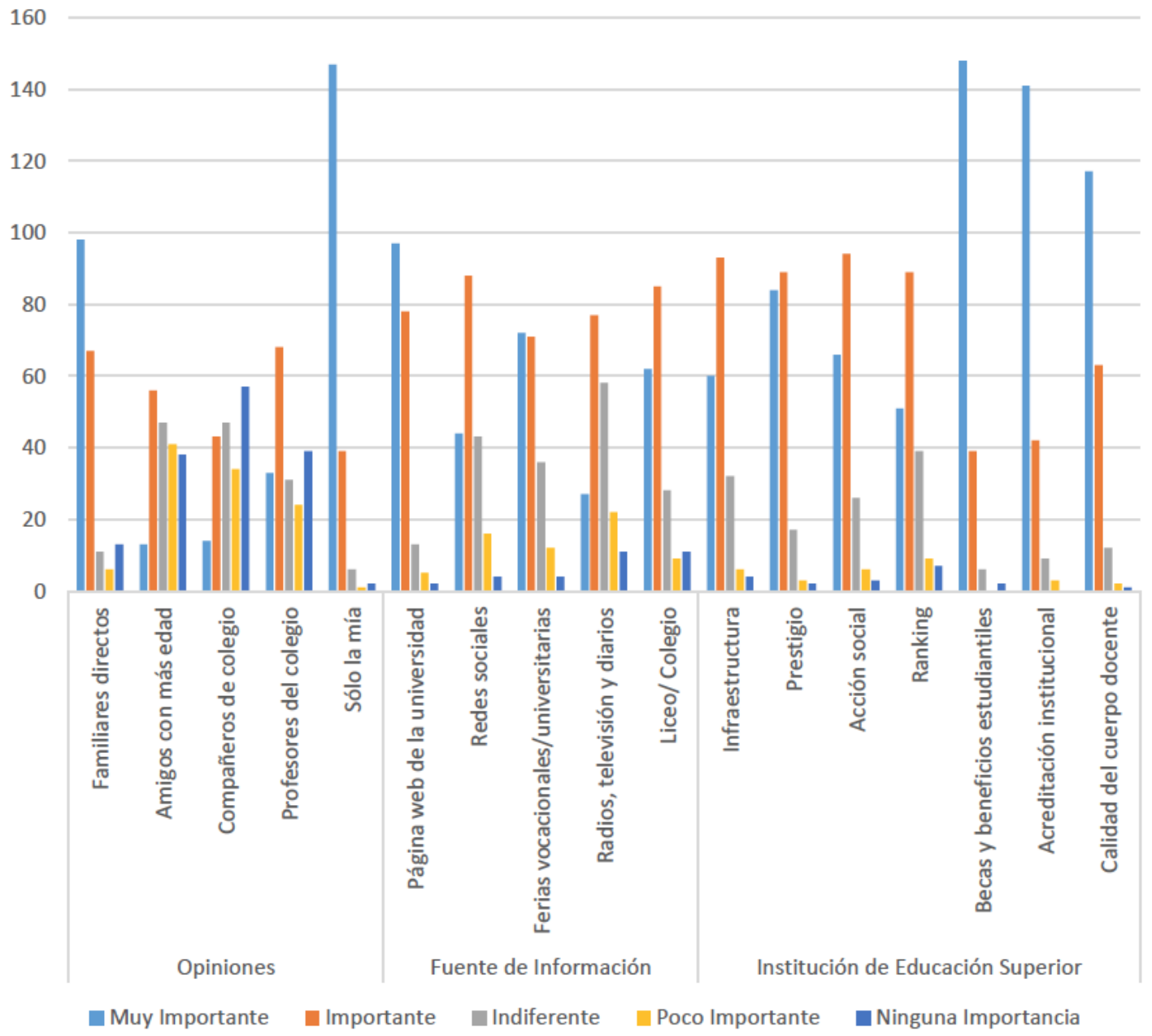

Figura 1. Aspectos importantes al momento de seleccionar una Institución de Educación Superior.

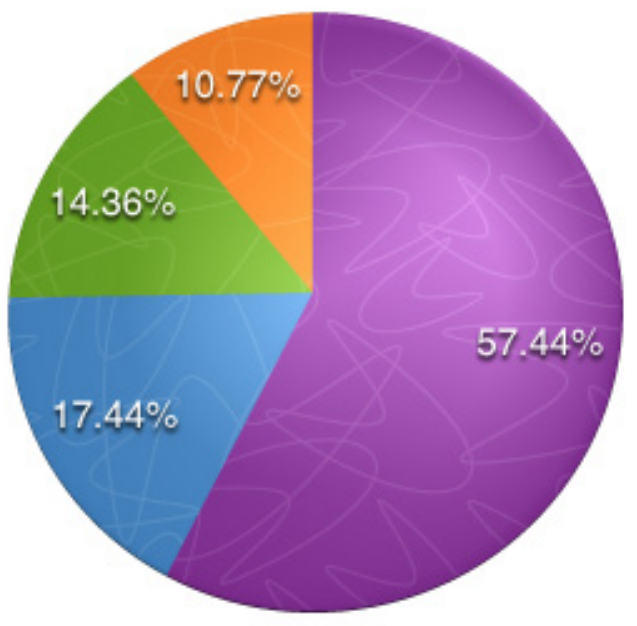

Ubicación - Acreditación Otros Carreras Disponibles

Figura 2. Motivos para la elección de la Universidad de Los Lagos. 


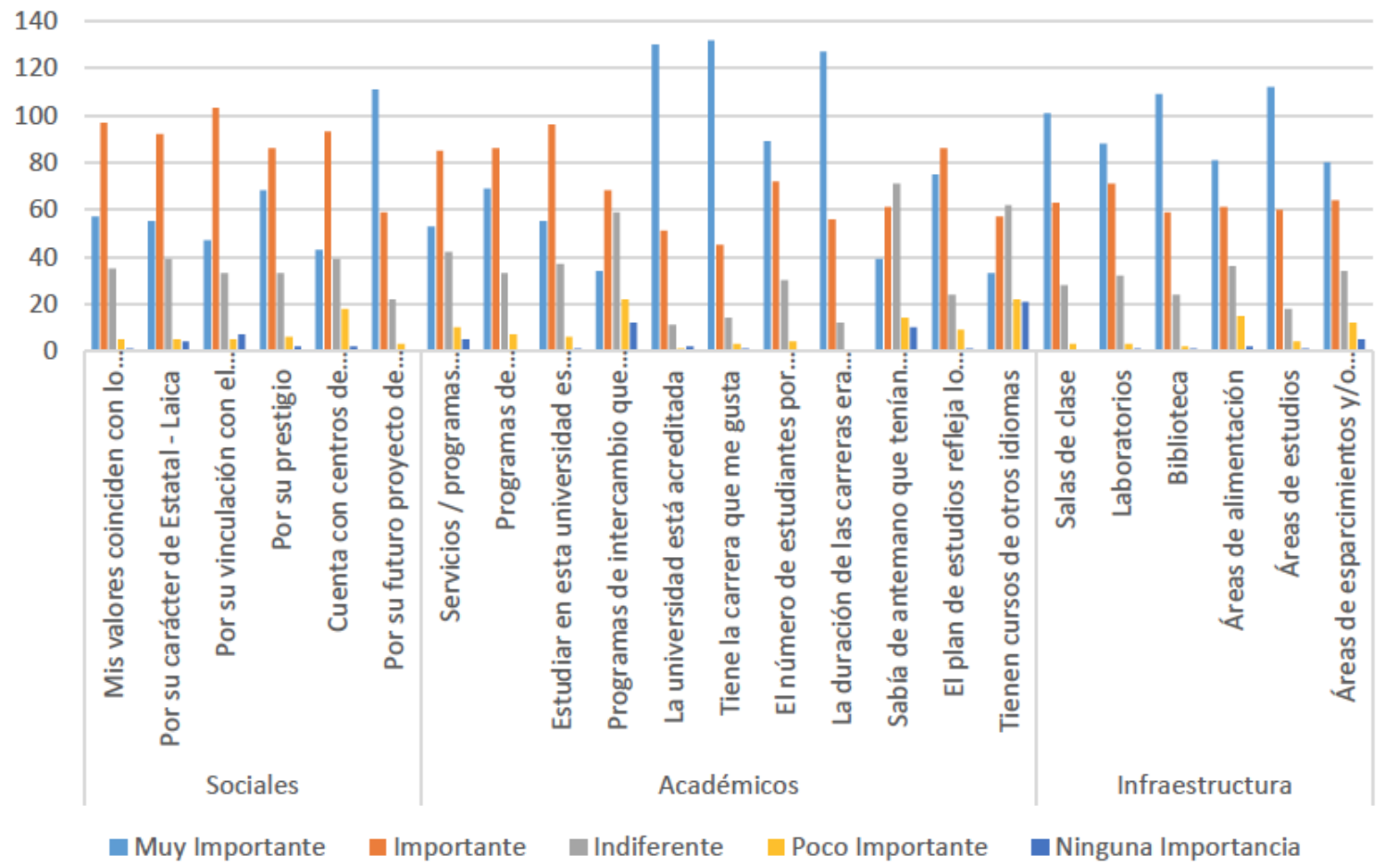

Figura 3. Principales aspectos de selección.

El proyecto de instalación de la Universidad en la sede Chiloé, Castro - Ancud sobresale como el principal aspecto de selección con un 56.92\%; seguido del prestigio que tiene en el territorio (34.87\%). Entre los aspectos académicos se destacan los factores de tener la carrera que se desea $(67.69 \%)$ y la acreditación institucional (66.67\%). Respecto a la infraestructura, el factor más importante para los estudiantes son las áreas de estudios con un $57.44 \%$, la existencia de una biblioteca (55.90\%); y las salas de clase propicias para el estudio (51.79\%).

\section{Selección de carrera a estudiar}

$\mathrm{Al}$ consultar a los estudiantes si la carrera en la cual están matriculados era su primera opción, un $71.79 \%$ respondió afirmativamente, mientras que un $28.21 \%$ de forma negativa.
A este último porcentaje se le consultó sobre cuál era su primera opción (ver tabla 2).

Al cuestionarles sobre cuál fue el principal motivo por el cual no se pudo acceder a la carrera, los estudiantes indicaron que la ubicación geográfica donde se encontraban las carreras deseadas $(30.91 \%)$ y los factores económicos (23.64\%), fueron los principales motivos. Otros elementos que influenciaron esa decisión fueron la vocación (20\%), la Prueba de Selección Universitaria (PSU) con un 10.91\%; y la empleabilidad con un $1.82 \%$, entre otros aspectos que no fueron especifica$\operatorname{dos}(12.73 \%)$. 
Tabla 2. Primera opción de carrera universitaria

\begin{tabular}{|c|c|c|}
\hline Variable & Categoría & Porcentaje \\
\hline & Ciencia y Tecnología & $30.91 \%$ \\
\cline { 2 - 3 } Área de co- & Educación & $27.27 \%$ \\
\cline { 2 - 3 } nocimiento & Salud & $20 \%$ \\
\cline { 2 - 3 } de la primera & Ciencias Sociales & 12.73 \\
\cline { 2 - 3 } opción & Actividad Física & $5.45 \%$ \\
\cline { 2 - 3 } & Otro & $1.82 \%$ \\
\cline { 2 - 3 } & No respondió & $1.82 \%$ \\
\hline
\end{tabular}

Selección de carrera matriculada en la Universidad de Los Lagos

Con respecto a los factores que se tuvieron en cuenta para matricularse en la carrera actual, se consultaron tres aspectos: económicos, sociales y académicos (ver figura 4).

Los aspectos económicos más importantes fueron las posibilidades de inserción laboral posterior a los estudios (64.10\%), seguidos de la continuidad de estos con un $61.03 \%$. En cuanto a los factores sociales, la vocación obtuvo un $68.72 \%$, y los valores de la carrera puntuaron con un $61.03 \%$. Finalmente, en los elementos académicos, se destacó la acreditación de la carrera (77.44\%), la duración de la misma (64.62\%); la calidad docente (64.10\%) y el plan de estudios (63.08\%).

\section{Discusión}

Esta investigación tuvo como propósito describir cuáles fueron los factores más importantes que tomaron en cuenta los estudiantes matriculados en la Universidad de Los Lagos

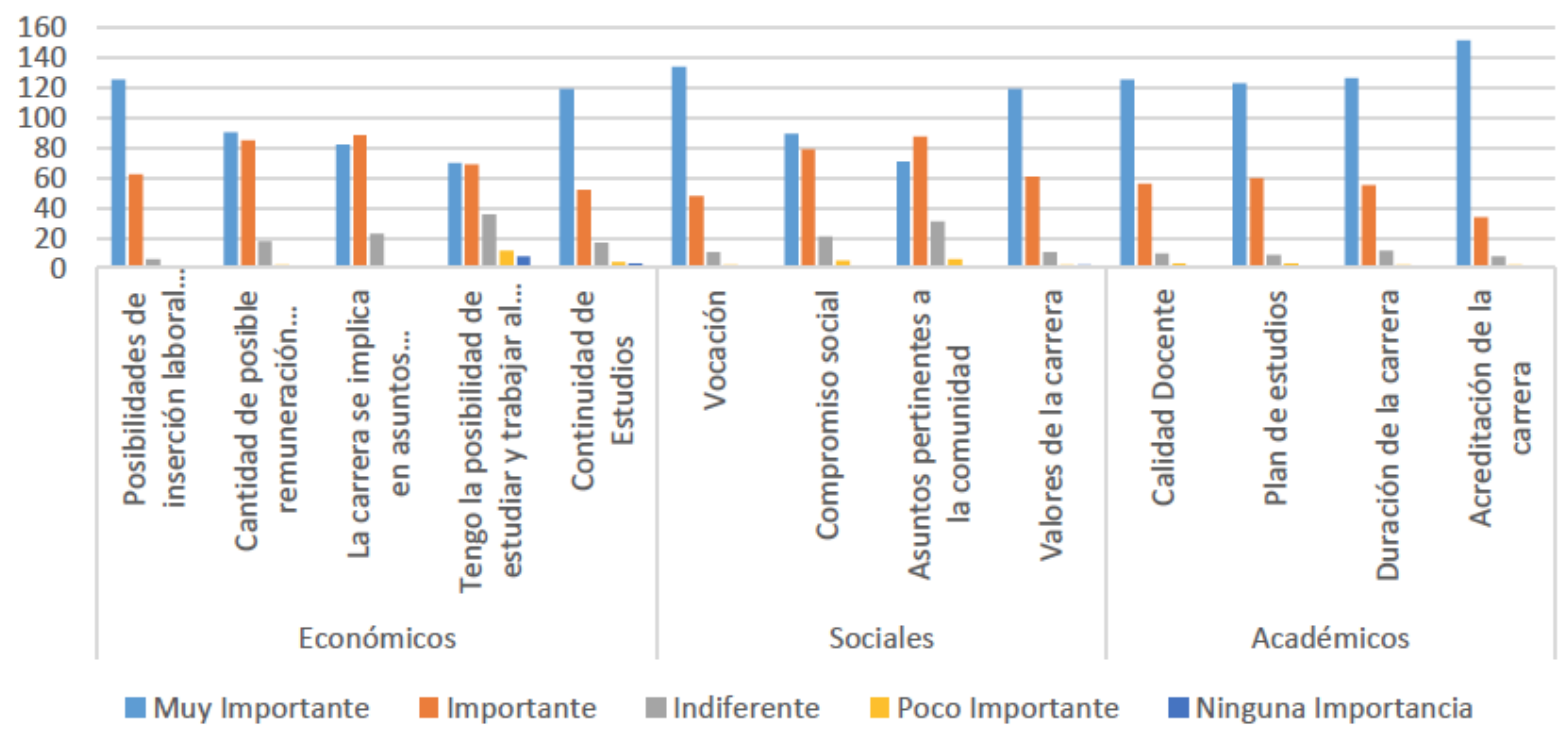

Figura 4. Aspectos importantes para selección de la carrera actual. 
de Chile en la sede Chiloé, Castro - Ancud de la cohorte 2019. Los resultados obtenidos muestran que, las becas y beneficios que una Universidad puede ofrecer $(75.90 \%)$ son el factor más importante al momento de seleccionar una institución de educación superior. Siendo de poca importancia el ranking universitario (3.59\%).

Aunque los estudiantes encuentran muy importante su opinión a la hora de seleccionar la Institución (75.38\%) y de poca importancia las opiniones de sus compañeros (29.23\%), un factor importante es el rol que cumple el/ la orientador/a en el establecimiento educacional. Es él/ella quien debe generar una estrategia para entregar la mayor información posible para que el estudiante pueda decidir.

Con respecto a las fuentes de información que utilizan los estudiantes para conocer las Instituciones de Educación Superior, el factor más importante fue la variable sitio web (49.74\%) y de poca importancia la información que se entrega a través de los medios de comunicación tradicionales (radios, televisión y diarios $(5.64 \%))$. Este factor es responsabilidad de las Instituciones, las cuales deben mantener actualizada la información necesaria para sus futuros estudiantes.

En cuanto a la selección de la Universidad de Los Lagos, el 54.44\% de los estudiantes escogió estudiar en esta institución por encontrarse cerca de su hogar, siendo que el 67.69\% de los estudiantes indicaron que en esta entidad se encontraba la carrera de su predilección. Aspectos como las salas de clase (51.79\%); exis- tencia de laboratorios (45.13\%), de biblioteca (55.90\%), áreas de alimentación (41.54\%), áreas de estudio (57.44\%) y áreas de esparcimiento y/o, ocio (41.03\%) también tuvieron parte dentro de la decisión. En esta dimensión cumplen una función muy importante las unidades de promoción y orientación de carreras, en las cuales se puede desarrollar un plan de acompañamiento para los establecimientos educacionales del territorio donde se encuentra la Universidad, a fin de conocer y vivenciar la vida universitaria.

$\mathrm{Al}$ analizar la selección de la carrera universitaria, el $71.79 \%$ de los estudiantes matriculados en la Universidad de Los Lagos, sede Chiloé, Castro - Ancud están cursando la carrera que ellos deseaban, a pesar de que un $28.21 \%$ tenían otra opción para estudiar. Dentro de estas opciones, sobresale la elección de una carrera profesional $(74.55 \%)$, especialmente en el área de ciencia y tecnología (30.91\%). Entre los principales factores para no acceder a las carreras sobresalen los aspectos de ubicación (30.91\%) y económicos (23.64\%). $\mathrm{Al}$ igual que en las dimensiones anteriores, es importante el rol que cumple el orientador y cómo esto se puede articular con las unidades de promoción u orientación de las Universidades para generar un plan de trabajo en conjunto y poder acompañar a los estudiantes al momento de seleccionar la carrera universitaria que desean.

Finalmente, se puede indicar que, al momento de seleccionar la carrera, tanto la inserción laboral una vez que el estudiante termina su carrera (64.10\%) como la vocación (68.72\%) son importantes. Entre los aspectos acadé- 
micos, no existió una diferencia significativa, pero se resaltan la calidad docente (64.10\%), el plan de estudios (63.08\%), la duración de la carrera $(64.62 \%)$ y la acreditación de la misma $(77.44 \%)$. En esta dimensión, es importante el rol que cumple la Universidad realizando una evaluación constante a las carreras que está impartiendo; tomando en cuenta la empleabilidad y la pertinencia que estas debieran tener en el territorio donde se encuentran insertas.
Con base en la literatura consultada y el contexto de Chiloé, se consideró importante tomar en cuenta que la elección de la universidad o de la carrera no necesariamente coincide con lo que el estudiante deseaba para sí. Al mismo tiempo, sí puede haber deseado la universidad elegida y no la carrera matriculada o viceversa.

\section{Referencias}

García A., J. y Moreno M., C. (2012) Factores considerados al seleccionar una universidad. Caso Ciudad Juárez. Revista Mexicana de Investigación Educativa, 17 (52), 287-305.

Hernández, R.; Fernández, C., y Baptista, P. (2010). Metodología de la Investigación. México: McGraw Hill Interamericana.

Mamani R., O. (2018). Factores que influyen en la elección de una universidad en los estudiantes jóvenes y adultos del Perú. Universidad Complutense de Madrid: Madrid.

Montesano, J.C. y Zambrano, E. (2013). Factores que influyen en la elección de una carrera universitaria en la Universidad Católica Andrés Bello. Universidad Católica Andrés Bello: Caracas.

Pérez B., J.M. (2017). Análisis de factores asociados a la elección de estudios universitarios utilizando técnicas de agrupamiento. Universitat Politécnica de Valencia: Valencia.

Slachevsky A., N. (2015). Una revolución neoliberal: la política educacional en Chile desde la dictadura militar. Educação e Pesquisa, 41(spe.), 1473-1486. https://doi.org/10.1590/S15179702201508141660

Torres, G. y Tamayo, M. (2018). Factores que determinan la elección de carrera profesional: en estudiantes de undécimo grado de colegios públicos y privados de Barrancabermeja. Revista Psicoespacios, 12 (20), 35-48. Universidad de Cudinamarca: Cudinamarca.

Villada, C. (2002). Factores asociados a la elección de la carrera y universidad. Grupo CHHES BIÓGENES, Universidad de Antioquía: Antioquía. 